\title{
Entropy-based detection of manoeuvring range-spread targets in homogeneous weather clutter
}

\author{
Shu-wen $\mathrm{Xu}^{*}$ and Peng-lang Shui
}

\begin{abstract}
This paper proposes a waveform-cross-entropy (WCEN)-based detection scheme to detect manoeuvring range-spread targets in homogeneous weather clutter. The input of the detector is composed of complex-valued high resolution range profiles (HRRPs) from a train of coherent pulses in the observation window. The observation window contains a detection window and a reference window, where the received data in reference window is clutter-only secondary data. The detector consists of temporal approximate whitening filtering for clutter suppression followed by the waveform-cross-entropy-based detection. The former is operated on the received vector at each range cell to whiten the clutter and enhance target returns. Then, the WCEN of each pair of filtered power-HRRPs is calculated to capture their sparsity, similarity and energy. The average of the WCENs of all the pairs is utilized as the test statistic to decide whether a target is present or not. Finally, we assess the proposed detector by the raw radar target data collected by high range resolution radar and simulated weather clutter. The experimental results show that the WCEN-based detector outperforms the existing detectors in detection performance for manoeuvring range-spread targets.
\end{abstract}

Keywords: Weather clutter, High resolution range profile (HRRP), Manoeuvring range-spread target, Waveform cross-entropy (WCEN)

\section{Introduction}

Wideband or ultra-wideband high range resolution radars (HRR) can spatially resolve a target into a number of scatterers distributed along the radial range. When target's size is larger than the range resolution cell of a HRR, the target occupies multiple contiguous range cells and is referred to as a range-spread target. For HRRs operating in clutter environments, the return at each range cell is the superimposition of the returns of possible target scatterers and a large number of clutter scatterers in the range cell. These returns distributed along the range cells form a complex high resolution range profile (HRRP) for each pulse. Range-spread target detection is based upon the received complex data matrix composed by the complex HRRPs collected from a train of coherent pulses. In this paper, we deal with high-speed manoeuvring range-spread target detection in ground-based

\footnotetext{
* Correspondence: swxu@mail.xidian.edu.cn

National Laboratory of Radar Signal Processing, Xidian University, Xi'an 710071, China
}

\section{Springer}

HRRs, where the background clutter is the weather clutter from the backscattering of clouds, rains or fogs.

During the past few decades, various methods have been developed for detecting range-spread targets. Early in 1971, range-spread target detection in white Gaussian noise was mentioned [1], where target's returns are assumed to be composed of non-empty range cells of Rayleigh distributed amplitudes and empty cells. Hughes [2] contrasted the two non-coherent detectors in white Gaussian noise, which integrates the energy of a single HRRP along the range cells by two different tactics, showing that their performances depend on the energy distribution of target returns in the HRRPs. In [3], with a known target range scattering function, the spectrum-matching detector is shown to be optimal among all non-coherent detectors. However, this assumption is difficult to be satisfied in practice. The methods mentioned above exploit the energy distribution of target HRRPs while not exploiting the waveform characteristics. The two recent non-coherent detectors in white Gaussian noise, the SSD-GLRT detector [4] and the MCOM detector [5], exploit the waveform characteristics. 
Compared with those detectors in white Gaussian noise, detecting range-spread targets in nonstationary clutter environments is more complicated and has recently received much more attentions [6-15]. Those methods mainly focus upon steady range-spread targets, such as air targets in steady flight or slow ship target in sea clutter. Complex HRRPs of steady range-spread targets can be modeled well and in this case coherent detectors outperform non-coherent ones. When target returns are assumed to be without range walking across cells during integration, the complex HRRPs to be integrated share an amplitude function along range cells, and the target returns of all the range cells share a Doppler shifts. The GLRT-based detectors developed in $[6,7]$ can effectively detect targets in homogenous clutter environments. In $[8,9]$, the order statistical sum of the generalized likelihood ratios at all the range cells of the detection window was recently developed for steady range-spread target detection in non-Gaussian clutter. The target return model available in [6-9] is unsuitable for range-spread targets in high-speed manoeuvring flight, whose complex HRRPs exhibit much more complicated change. The return model with both range and Doppler spreads can describe targets with rotation but without range walking across cells during integration [10-12]. Under this model, the GLRT-based detectors suffer from additional performance loss, because the rotation incurs different Doppler shifts in the individual cells and too many model parameters need to be estimated from the received data corrupted by strong clutter. Also, the spatially adaptive methods [13-15] were proposed for radars using antenna arrays to detect range-spread targets.

With fast development of modern martial technique, aircrafts possess higher speed and better maneuverability. It is an inevitable problem in HRRs to detect highspeed maneuvering targets in clutter environments. Because of the target's high-speed maneuvering, the target may move across several range cells from one pulse to another, which results in a time delay term in the range profiles for two or more adjacent pulses, namely, range walking. Even in a short integration interval, the HRR's returns of such a target suffer from range walking across cells and Doppler spread from target rotation $[16,17]$. The simpler models mentioned previously are not enough to model their returns. The $2 \mathrm{D}$ scattering center model $[18,19]$ in the ISAR imaging and automatic target recognition (ATR) can model the target returns well. However, the GLRT-based detector using the 2D model is difficult to efficiently detect high-speed manoeuvring range-spread targets, because too many parameters have to be estimated from the received data of low SNR or SCR. In our previous work [5,20], the two heuristic detectors, exploiting the features of targets HRRPs rather than rigorous parametric models of target returns, were developed in white Gaussian noise. In [21], based on the diversity between the information entropy of target echo and disturbance, the detection algorithm of single pulse for range spread target via Renyis entropy is proposed. Then, we proposed a detector based on waveform entropy of the arithmetic average of multiple successive high-resolution range profiles (HRRP) in [22]. In [22], the detector only consider the sparse character of the arithmetic average combined HRRP, which hasn't consider the energy character and the cross-correlation of the HRRPs in the integrated pulses. Here, a new detector based upon temporal whitening filters and waveform cross-entropy (WCEN) is proposed to detect high-speed manoeuvring range-spread targets in weather clutter.

This paper is organized as follows. Section 2 describes the detection problem and gives a full analysis on target returns of high-speed manoeuvring range-spread targets by raw radar data. The waveform-cross-entropy-based detector is presented in Section 3. Section 4 reports the experimental results and the proposed detector is compared with the MCOM detector and GLRT detector. Finally, we conclude our paper in Section 5.

\section{Detection problem description of high-speed manoeuvring range-spread targets}

\subsection{Detection problem description}

Assume that a wideband HRR operates in the scan mode and transmits $N$ coherent pulses at each beam position. From the $N$ coherent pulses and at $M+K$ contiguous range cells, the radar received data is a $2 \mathrm{D}$ array $\mathbf{z}(m, n)$, where $m$ labels the range cells and $n$ denotes the pulses. We consider a clutter-dominant scenario with a neglectable receiver noise. Thus, the received data is either clutter or target returns plus clutter. The detection aims at judging whether a target is present or absent from the received data. The received vector, clutter vector and target return vector at the $m$-th range cell and $n$-th pulse, are represented by

$$
\begin{aligned}
\mathbf{z}_{m} & =[z(m, 0), z(m, 1), \ldots, z(m, N-1)]^{T}, \\
\mathbf{Z}_{n} & =[z(0, n), z(1, n), \ldots, z(M-1, n)]^{T}, \\
\mathbf{c}_{m} & =[c(m, 0), c(m, 1), \ldots, c(m, N-1)]^{T}, \\
\mathbf{C}_{n} & =[c(0, n), c(1, n), \ldots, c(M-1, n)]^{T}, \\
\mathbf{s}_{m} & =[s(m, 0), s(m, 1), \ldots, s(m, N-1)]^{T}, \\
\mathbf{S}_{n} & =[s(0, n), s(1, n), \ldots, s(M-1, n)]^{T},
\end{aligned}
$$

The first $M$ contiguous range cells form the detection window and in which the data is referred to as the primary data. The latter $K$ range cells, called as the secondary data, are around the detection window. The secondary data is assumed to be clutter-only. Moreover, 
the clutter vectors in the primary and secondary data are assumed to share a same temporal covariance matrix. The detection window and the $K$ reference range cells form an observation window with $M+K$ range cells. As a result, the range-spread target detection in clutter boils down to the following binary hypothesis testing [23]:

$$
\left\{\begin{array}{c}
\mathrm{H}_{0}: \mathbf{z}_{m}=\mathbf{c}_{m}, m=0,1, \ldots ., M-1, M, \ldots, M+K-1 . \\
\mathrm{H}_{1}:\left\{\begin{array}{c}
\mathbf{z}_{m}=\mathbf{s}_{m}+\mathbf{c}_{m}, \quad m=0,1, \ldots ., M-1 \\
\mathbf{z}_{m}=\mathbf{c}_{m}, \quad m=M, \ldots, M+K-1 .
\end{array}\right.
\end{array}\right.
$$

Assume that the HRR operates in the scan mode, meaning that the number $N$ of the pulses at each beam position is not very many, typically, $N \leq 32$. Different from the ground clutter and sea clutter, the weather clutter has a long homogenous interval relative to the range resolution of a HRR. For the detection problem in (2), we have the following basic assumptions: 1) In the observation window, the clutter is homogenous and can be modeled by the SIRV [9] with complex Gaussian distribution, meaning that the clutter vectors in all the range cells of the observation window are independent identical distributed (i.i.d) complex Gaussian random vectors. 2) In the observation window, the clutter vectors have the probability density function (PDF) as follows:

$$
p\left(\mathbf{c}_{m}\right)=\frac{1}{\pi^{N} \operatorname{det} \mathbf{R}} \exp \left(-\mathbf{c}_{m}^{H} \mathbf{R}^{-1} \mathbf{c}_{m}\right),
$$

where the temporal covariance matrix is unknown and varying with observation windows. Note that these two assumptions are unsuitable for detection of targets located near the boundary of homogenous regions of the clutter, for instance, near the borders of clouds. It can be seen that the detection problem (2) is equivalent to target detection in colored Gaussian clutter with unknown covariance matrix in each observation window. The clutter power, corresponding to the texture component in the SIRV model, is varying with observation windows and its distribution impacts the detection performance.

\subsection{HRRPs of high-speed manoeuvring targets}

In the HRRP-based target recognition and ISAR imaging $[16,17]$, the return characteristics of range-spread targets have been thorough investigated and it is shown that the $2 \mathrm{D}$ scattering center model parameterize target returns well. However, too many model parameters make the $2 \mathrm{D}$ model unsuitable for detection of range-spread targets, particularly, for detection of high-speed manoeuvring ones in strong noise or clutter. Because the 2D scattering model has too many parameters, several models with a smaller number of parameters are developed for range-spread target detection in noise or clutter environments.

When targets are without range walking across cells and the Doppler spread can be negligible during integration, target return vectors are modeled as the tensor of a complex HRRP and a Doppler steering vector [6]:

$$
\begin{aligned}
& s(m, n)=a(m) \exp \left(2 \pi j n T_{r} f_{d}\right), \\
& m=0,1, \cdots, M-1, n=0,1, \cdots, N-1,
\end{aligned}
$$

where $a(m), m=0,1, \cdots, M-1$ is a complex HRRP, $T_{r}$ is the pulse repetition interval, and $f_{d}$ is the Doppler shift. The model (4) is suitable for the cases that the range resolution is not too high, target is low-speed and steady flight, and the integration interval is not too long. When target rotation can't be neglected and some range cells probably contain a few strong scatterers but target has not range walking across cells during integration, the $2 \mathrm{D}$ scattering center model has to be employed and target return vectors are as follows [10]:

$$
s(m, n)=\sum_{k=1}^{p_{m}} a(m, k) \exp \left(2 \pi j n T_{r} f_{m, k}\right)
$$

where the $m$-th cell contains $p_{\mathrm{m}}$ strong scatterers and their Doppler shifts are different due to their different distances away from the radar along the LOS of the radar. More generally, when the range walking across cells are also considered, the 2D scattering center model for the ISAR imaging must be used, and target returns are written as follows [19]:

$$
s(m, n)=\sum_{k=1}^{p_{m}} a(m-\tau(n), k) \exp \left(2 \pi j n T_{r} f_{m-\tau(n), k}\right),
$$

where $\tau(n)$ is the range walking from the first pulse to the $n$-th pulse. When the GLRT-based detection schemes are used for the three return models, the models (4), (5) and (6) have $M+1,2 \sum_{k=1}^{M} p_{m}$ and $2 \sum_{k=1}^{M} p_{m}+N$ parameters that must be estimated from $M \times N$ received data, respectively. In the cases of low SNR or SCR, the GLRT-based detectors using the models (5) and (6) suffer from severe performance loss because of too many parameters to be estimated.

Here, the measured data is collected in a good weather condition and has quite high SCR. The HRR has a bandwidth $150 \mathrm{MHz}$, range resolution 1 meter, and the pulse repetition frequency (PRF) $500 \mathrm{~Hz}$. The data is regarded to be free of clutter. In Figure 1a, we show the amplitudes of the returns in 500 contiguous range cells and from 3000 
(a) Amplitudes of target echoes

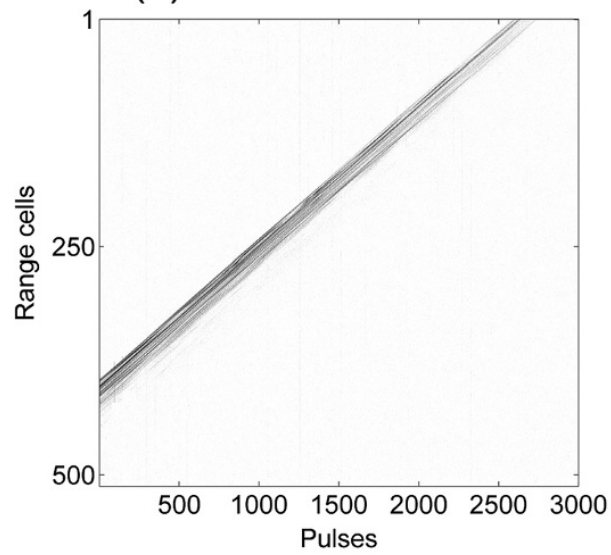

(b) Comparison of two amplitude-HRRPs

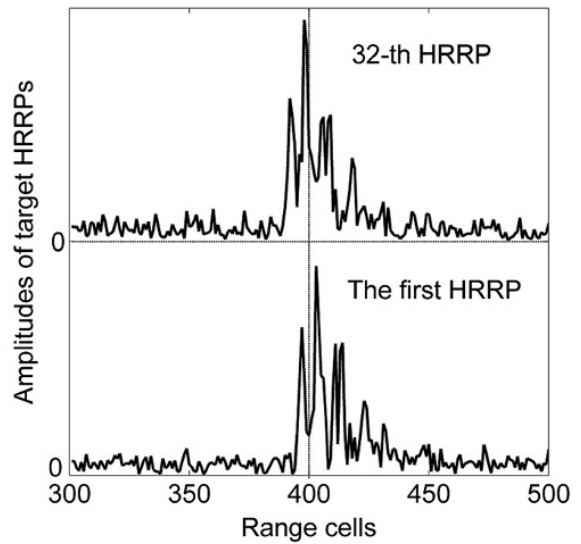

Figure 1 Amplitudes of target returns in the raw radar data and the similarity of two amplitude-HRRPs.

successive pulses, where each 32 successive pulses are coherent. Target returns suffer from obvious range walking across cells over a range cell in six successive pulses. In Figure 1b, the first and 32-th amplitude-HRRPs are plotted. It is worthy to note that strong scattering cells in the HRRPs are sparse and the two amplitude-HRRPs have a similar waveform except a range walking. Because the phase information is quite difficult to be utilized [5], we propose a new waveform cross entropy based detector using the amplitude HRRP to detect the manoeuvring range spread targets.

\section{Waveform-cross-entropy-based detector of manoeuvring range-spread targets}

3.1. Approximate whitening filter along pulses

In terms of the basic assumptions on the detection problem (2), the weather clutter is homogenous in the observation window and all the clutter vectors at the range cells in the observation window are i.i.d Gaussian random vectors with an unknown covariance matrix and the number $K$ of the reference range cells is much more than the dimension $N$ of the clutter vectors. The temporal covariance matrix of the clutter vector in the primary data can be estimated from the clutter-only secondary data by the sample covariance matrix (SCM) estimator [24]:

$$
\hat{\mathbf{R}}=\frac{1}{K} \sum_{k=1}^{K} \mathbf{z}_{M+k}^{H} \mathbf{z}_{M+k}
$$

where $\hat{\mathbf{R}}$ is a positive-definite Hermitian matrix. The estimate precision depends on the ratio $K / N$ and the estimate $\hat{\mathbf{R}}$ tends toward the actual covariance matrix $\mathbf{R}$ as the ratio $K / N$ tends toward infinite [10]. In term of the estimate $\hat{\mathbf{R}}$, the temporal whitening filtering is row-wise realized by:

$$
\left\{\begin{aligned}
H_{0}: \tilde{\mathbf{z}}_{m} & =\mathbf{z}_{m} \hat{\mathbf{R}}^{-1 / 2}=\mathbf{c}_{m} \hat{\mathbf{R}}^{-1 / 2}=\tilde{\mathbf{c}}_{m} \\
H_{1}: \tilde{\mathbf{z}}_{m} & =\mathbf{z}_{m} \hat{\mathbf{R}}^{-1 / 2}=\left(\mathbf{s}_{m}+\mathbf{c}_{m}\right) \hat{\mathbf{R}}^{-1 / 2}=\mathbf{s}_{m} \hat{\mathbf{R}}^{-1 / 2} \\
& \left.+\mathbf{c}_{m} \hat{\mathbf{R}}^{-1 / 2}=\tilde{\mathbf{s}}_{m}+\tilde{\mathbf{c}}_{m}\right), m=0,1, \cdots, M-1
\end{aligned}\right.
$$

Because $K \gg N$ and $\hat{\mathbf{R}}$ is sufficiently close to $\mathbf{R}$, the whitened clutter vectors $\tilde{\mathbf{c}}_{m}$ can be regarded to be zeromean white complex Gaussian vectors with the identity covariance matrix for the sake of convenience in analysis. Moreover, the clutter vectors at different range cells are mutually independent. As a result, the clutter array $\tilde{\mathbf{c}}(m, n), m=0,1, \cdots, M-1, n=0,1, \cdots, N-1$ is a 2D zero-mean white complex Gaussian random matrix with the identity covariance. The whitening filtering enhances target returns but the extent of enhancement is relevant to the Doppler shifts of target returns at individual range cells.

After the temporal whitening filtering, target detection is reduced to the following binary hypothesis testing:

$$
\left\{\begin{array}{l}
\mathrm{H}_{0}: \tilde{\mathbf{Z}}_{n}=\tilde{\mathbf{C}}_{n}, n=0,1, \cdots, N-1, \\
\mathrm{H}_{1}: \tilde{\mathbf{Z}}_{n}=\tilde{\mathbf{S}}_{n}+\tilde{\mathbf{C}}_{n}, n=0,1, \cdots, N-1
\end{array}\right.
$$

where $\quad \tilde{\mathbf{S}}_{n}=[s(0, n), s(1, n), \cdots, s(M-1, n)], n=0,1, \cdots, N-1$, are the filtered target returns and $\tilde{\mathbf{C}}_{n}=[c(0, n), c(1, n), \cdots$, $c(M-1, n)], n=0,1, \cdots, N-1$, are the whitened clutter. Withdrawing the phase information, the power-HRRPs are used for detection and the problem boils down to the 
binary hypothesis testing:

$$
\left\{\begin{array}{l}
\mathrm{H}_{0}: \bar{z}(m, n)=|\tilde{c}(m, n)|^{2} \\
\mathrm{H}_{1}: \bar{z}(m, n)=|\tilde{s}(m, n)+\tilde{c}(m, n)|^{2}, \\
\quad m=0,1, \cdots, M-1 ; n=0,1, \cdots, N-1 .
\end{array}\right.
$$

Under the $H_{0}$ hypothesis, $\bar{z}(m, n)$ obey i.i.d single-side exponent distribution:

$$
p\left(x=\bar{z}(m, n) \mid H_{0}\right)=\left\{\begin{array}{cc}
e^{-x}, x \geq 0 \\
0, & x<0
\end{array} .\right.
$$

Under the $H_{1}$ hypothesis, because of nonlinear modulus square, no simple parametric expressions are suitable for the filtered target returns $\tilde{\mathbf{s}}(m, n)$ and thus no simple model is for $\bar{z}(m, n)$. Then, we exploit the three inherent features of target returns $\tilde{\mathbf{s}}(m, n)$ : sparsity, similarity and high energy, to construct a new detector.

\subsection{Waveform cross-entropy of power-HRRPs}

In order to characterize the sparsity and similarity of power-HRRPs, the entropy concept of the vector is exploited. The vector entropy originated from the Shannon information entropy $[25,26]$ can measure the sparsity of a vector. Let $x(n), n=0,1, \cdots, N-1$ be an $N$-dimensional complex or real vector, its vector entropy is defined as:

$$
\begin{aligned}
& \varepsilon(x) \equiv-\sum_{n=0}^{N-1} p(n) \ln (p(n)), \\
& p(n)=|x(n)|^{2} /\|x\|_{2}^{2}
\end{aligned}
$$

where $p(\mathrm{n})$ is an energy distribution of the vector at individual components. When its energy is concentrated on a small number of components, the entropy takes a small value; otherwise, it takes a large value. Hence, the less the vector entropy is, the sparser the large components in the vector are.

Here, we need to introduce a figure of merit to measure both the sparsity and similarity of two power-HRRPs. In terms of the previous analysis, target power-HRRPs collected from two successive pulses share a similar waveform. Strictly speaking, their strong scattering cells often appear at the same positions except an unknown range walking. The maximally possible range walking depends on the radar range resolution, the maximally possible radial velocity of the target, and the pulse repetition interval (PRI) of the radar. Let the range resolution of the radar be $\delta r$, the maximal possible radial velocity be $v_{\max }$, and the PRI of the radar be $T_{r}$. The maximal possible range walking across cells between the $n_{i}$-th pulse and the $n_{j}$-th pulse is:

$$
\Delta\left(n_{i}, n_{j}\right)=f i x\left(\frac{\nu_{\max } T_{r}\left|n_{j}-n_{i}\right|}{\delta r}\right)
$$

where $f i x(\mathrm{x})$ denotes the integer nearest to $x$. For two power-HRRPs, the range walking can be estimated by the sliding correlation [19], i.e., the range walking $\bar{\tau}_{i j}$ is calculated by

$$
\bar{\tau}_{i j}=\underset{|\tau| \leq \Delta\left(n_{i}, n_{j}\right)}{\arg \max }\left\{\sum_{m=0}^{M-1} \bar{z}\left(m, n_{i}\right) \bar{z}\left(m-\tau, n_{j}\right)\right\}
$$

where $\bar{z}\left(m-\tau, n_{j}\right)=\bar{z}\left(\tau-m-1, n_{j}\right)$ when $m-\tau<0$, and $\bar{z}\left(m-\tau, n_{j}\right)=\bar{z}\left(2 M-(m-\tau)-1, n_{j}\right)$ when $m-\tau>M-1$. Further, we construct a new vector from the two power-HRRPs, defined by

$$
y_{n_{i}, n_{j}}(m) \equiv \bar{z}\left(m, n_{i}\right) \bar{z}\left(m-\bar{\tau}_{i j}, n_{j}\right)
$$

where $\bar{z}\left(m-\bar{\tau}_{i j}, n_{j}\right)=\bar{z}\left(\bar{\tau}_{i j}-m-1, n_{j}\right)$ when $m-\bar{\tau}_{i j}<0$, and $\bar{z}\left(m-\bar{\tau}_{i j}, n_{j}\right)=\bar{z}\left(2 M-\left(m-\bar{\tau}_{i j}\right)-1, n_{j}\right)$ when $m-\bar{\tau}_{i j}>M-1$. When the two power-HRRPs are both sparse and similar, $y_{n_{i}, n_{j}}(m)$ is also sparser, because the strong scattering cells in a power-HRRP are always multiplied by the strong scattering cells at the other power-HRRPs. As a result, the vector entropy of $y_{n_{i}, n_{j}}(m)$ is

$$
\begin{aligned}
\varepsilon\left(\mathbf{y}_{n_{i}, n_{j}}\right) & =-\sum_{m=0}^{M-1} p_{n_{i}, n_{j}}(m) \ln p_{n_{i}, n_{j}}(m) \\
p_{n_{i}, n_{j}}(m) & =\frac{y_{n_{i}, n_{j}}^{2}(m)}{\left\|\mathbf{y}_{n_{i}, n_{j}}\right\|_{2}^{2}} .
\end{aligned}
$$

where the vector entropy reflects the sparsity and the similarity of the two power-HRRPs. Due to the normalization in the second equation of (17), $\varepsilon\left(\mathbf{y}_{n_{i}, n_{j}}\right)$ is independent of the energy of the two power-HRRPs. The energy of returns is an indispensable feature in radar target detection. Combining the energy feature with the vector entropy in (17), we construct the quality with the following form

$$
\lambda\left(n_{i}, n_{j}\right) \equiv \frac{\varepsilon\left(\mathbf{y}_{n_{i}, n_{j}}\right)}{\left\|\overline{\mathbf{Z}}_{n_{i}}\right\|_{1}\left\|\overline{\mathbf{Z}}_{n_{j}}\right\|_{1}} .
$$

to reflect the sparsity, similarity, and energy of the two power-HRRPs, where $\overline{\mathbf{Z}}_{n_{i}}=\left[\bar{z}\left(0, n_{i}\right), \bar{z}\left(1, n_{i}\right), \cdots, \bar{z}\left(M-1, n_{i}\right)\right], \overline{\mathbf{Z}}_{n_{j}}$ $=\left[\bar{z}\left(0, n_{j}\right), \bar{z}\left(1, n_{j}\right), \cdots \bar{z}\left(M-1, n_{j}\right)\right]$. We refer to the quality in (18) as the waveform cross-entropy (WCEN) of the two power-HRRPs. In terms of the definition (18), the WCEN takes a small value when the two power-HRRPs are sparse and similar and of high energy, and takes a large value when they are neither sparse nor similar. In other words, the WCENs of different pairs of power- 
HRRPs take small values when a target is present while take large values when no target is present. It must be noted that the WCEN $\lambda\left(n_{i}, n_{i}\right)$ reflects the sparsity and energy of the $n_{i}$-th power-HRRP. Moreover, because $\lambda\left(n_{i}, n_{j}\right)=\lambda\left(n_{j}, n_{i}\right)$, there are $N(N+1) / 2$ WCENs from $N$ power-HRRPs that can be utilized to judge whether a target is present or not.

\subsection{Test statistic and waveform-cross-entropy-based detector}

From $N$ power-HRRPs, we construct a test statistic as follows:

$$
\xi \equiv \frac{2}{N(N+1)} \sum_{n_{i}=0}^{N-1} \sum_{n_{j}=n_{i}}^{N-1} \lambda\left(n_{i}, n_{j}\right)
$$

It is the arithmetic average of the $N(N+1) / 2$ WCENs. In fact, other weighted average can also be used for detection. The experimental results to raw target data show that the weighting does not bring obvious improvement in detection performance. Thus, the simple test statistic in (19) is used in this paper. Here, the detection scheme is shown in Figure 2, where $\mathbf{z}_{m}$ denotes the received vectors of length $\mathrm{N}$ at the $\mathrm{m}$-th range cell, $\tilde{\mathbf{Z}}_{n}$ denotes the whitened filtered HRRP of length $M$ at the n-th pulse, $\overline{\mathbf{Z}}_{n}$ denotes the whitened filtered powerHRRP of length $M$ at the $n$-th pulse, $\mathbf{y}_{n_{i}, n_{j}}$ denotes the joint probability vector constructed from the $n_{i}$-th power-HRRP and $n_{j}$-th power-HRRP, and $\lambda\left(n_{i}, n_{j}\right)$ denotes the Waveform entropy of the $n_{i}$-th power-HRRP and $n_{j}$-th power-HRRP.
Based upon the test statistic, the decision is carried out by.

$$
\left\{\begin{array}{c}
\xi \leq \eta, \text { a target is present, } \\
\xi>\eta, \text { no target is present, }
\end{array}\right.
$$

where $\eta$ is the decision threshold. For a given false alarm probability, determining the decision threshold needs to know the conditional probability density function $p\left(\xi \mid H_{0}\right)$. However, it can't be analytically obtained because of a large amount of nonlinear operations in calculation of the WCENs. In fact, this is a common problem in nonparametric detectors. In this case, the decision thresholds have to be determined by Monte-Carlo tests to clutter-only data and the performance evaluations have to be performed by the experimental results to real data rather than rigorous theoretical analysis [27].

When the received vectors are clutter-only, the test statistic is the arithmetic average of $N(N+1) / 2$ positive random variables. Though their distributions are unobtainable, they are all of finite means and variances. As $N$ is large enough, $p\left(\xi \mid H_{0}\right)$ approximates to the normal distribution $\mathbf{N}\left(u, \sigma^{2}\right)$ with mean $u$ and variance $\sigma^{2}$, which can be estimated from a relative small number of the Monte-Carlo tests to clutteronly data. For example, when $N=4,8,16$ and $32, v_{\max } \underline{T}_{r} / \delta r$ $=0.5, M=128$ and $K=512$, the weather clutter is a narrowband process and thus its covariance matrix is assumed as the form $[\mathbf{R}]_{i j}=\rho^{|i-j|}, 1 \leq i, j \leq N$, where $\rho$ is one-lag correlation coefficient the test statistic $\xi$ under the $H_{0}$ hypothesis have the histograms and their normal fitting curves, as shown in Figure 3. Then in Figure 4, we shown the curves of function $1-F\left(\xi \mid H_{0}\right)$ computed from the empirical cumulative distribution function (CDF) $F\left(\xi \mid H_{0}\right)$ and their normal

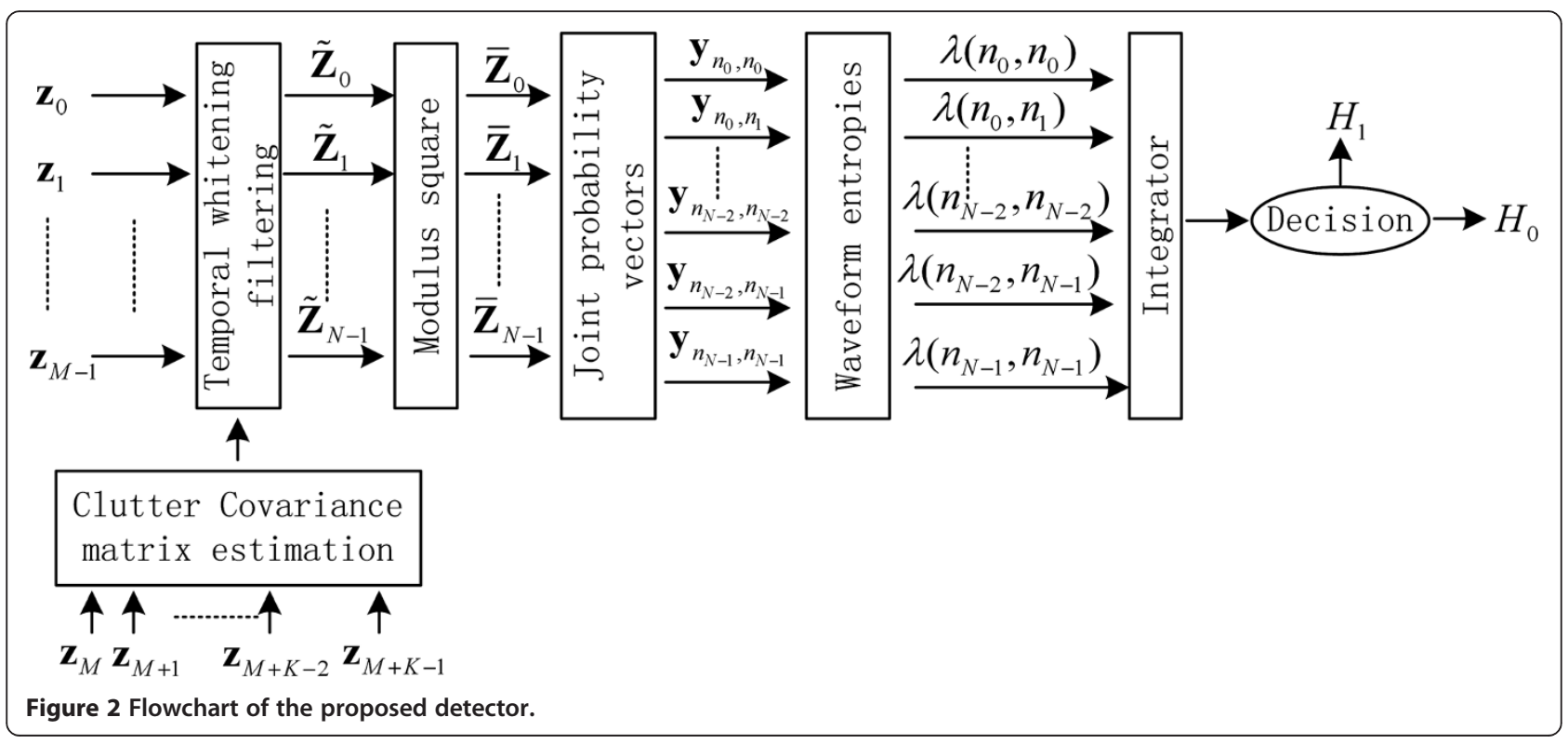



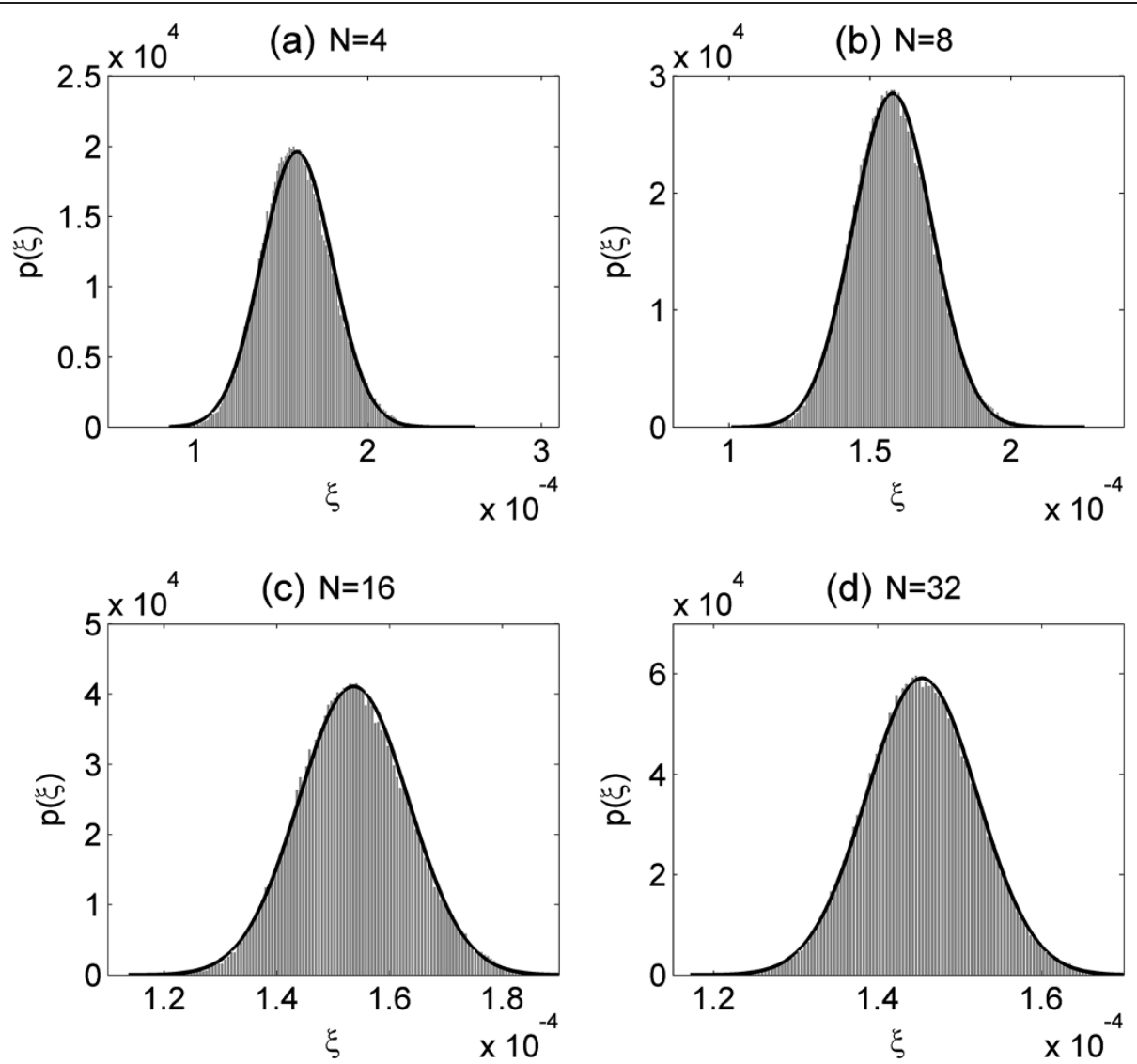

Figure 3 Histograms of the test statistic under the $\mathrm{H}_{0}$ hypothesis and their normal fitting curves.

fitting CDF, where the gray bars denote the histograms and the black curves are their normal fitting curves and $\rho=0.9$.

It can be seen from Figures 3 and $4, p\left(\xi \mid H_{0}\right)$ and $F\left(\xi \mid H_{0}\right)$ with $\mathrm{N}=8,16$ and 32 can be fitted well by the probability density function (PDF) and cumulative distribution function (CDF) from normal distributions, respectively. While that with $\mathrm{N}=4$ is fitted by the normal distribution with a slight departure. When $\mathrm{N} \geq 8$, the detection statistic is the average of more than $\mathrm{N}(\mathrm{N}+1) / 2$ random variables. Through the approximate whitening filter, the whitened clutter vectors $\tilde{\mathbf{c}}_{m}$ can be regarded to be zero-mean white complex Gaussian vectors with the identity covariance matrix approximately. Moreover, the clutter vectors at different range cells are mutually independent. As a result, the clutter array $\tilde{\mathbf{c}}(m, n)$ is a $2 \mathrm{D}$ zero-mean white complex Gaussian random matrix with the identity covariance. Therefore, in the $\mathrm{H}_{0}$ hypothesis, $\lambda\left(n_{i}, n_{j}\right)\left(n_{i}=0,1, \cdots, N-1\right.$, $\left.n_{j}=0,1, \cdots, N-1\right)$ are mutually independent, and each $\lambda\left(n_{i}, n_{j}\right)$ has limited mathematical expectation and variance. In terms of the central-limit theorem, when $\mathrm{N}$ is large enough, the detection statistic $\xi$ in the $\mathrm{H}_{0}$ hypothesis approximately obeys normal distribution, which can be verified by the experiments in Figures 3 and 4 . Thus, $\mathrm{p}(\xi \mid$ $H_{0}$ ) with $\mathrm{N} \geq 8$ are assumed to obey normal distributions whose means $u$ and variances $\sigma^{2}$ are estimated from a relatively small number of the Monte-Carlo tests to clutteronly data at the given parameters of the detector. When $\mathrm{N}$ $<8$, the decision thresholds have to be determined by the histograms from a large number of Monte-Carlo tests to clutter-only data. Generally, the number of the MonteCarlo tests should be not less than $100 / \mathrm{pf}$, where $p_{f}$ is the false alarm probability. For $\mathrm{N} \geq 8$, the decision threshold at a false alarm probability $p_{f}$, is determined by

$$
\eta=u+\sigma \Phi^{-1}\left(1-p_{f}\right)
$$

where $\Phi(x)$ is the cumulative distribution function of zeromean unit-variance normal random variable and $\Phi^{-1}$ is its inverse function. $u$ and $\sigma$ are the fitting mean and standard deviation, respectively.

When the reference range cells available are many enough relative to the dimension of the clutter vectors, the whitened clutter vectors are quite close to the i.i.d zeromean complex Gaussian random vectors with the identity covariance matrix, independent of the power and covariance of the original clutter. In this way, under a given decision threshold, the proposed detector achieves an approximate constant false alarm ratio (CFAR) with respect 


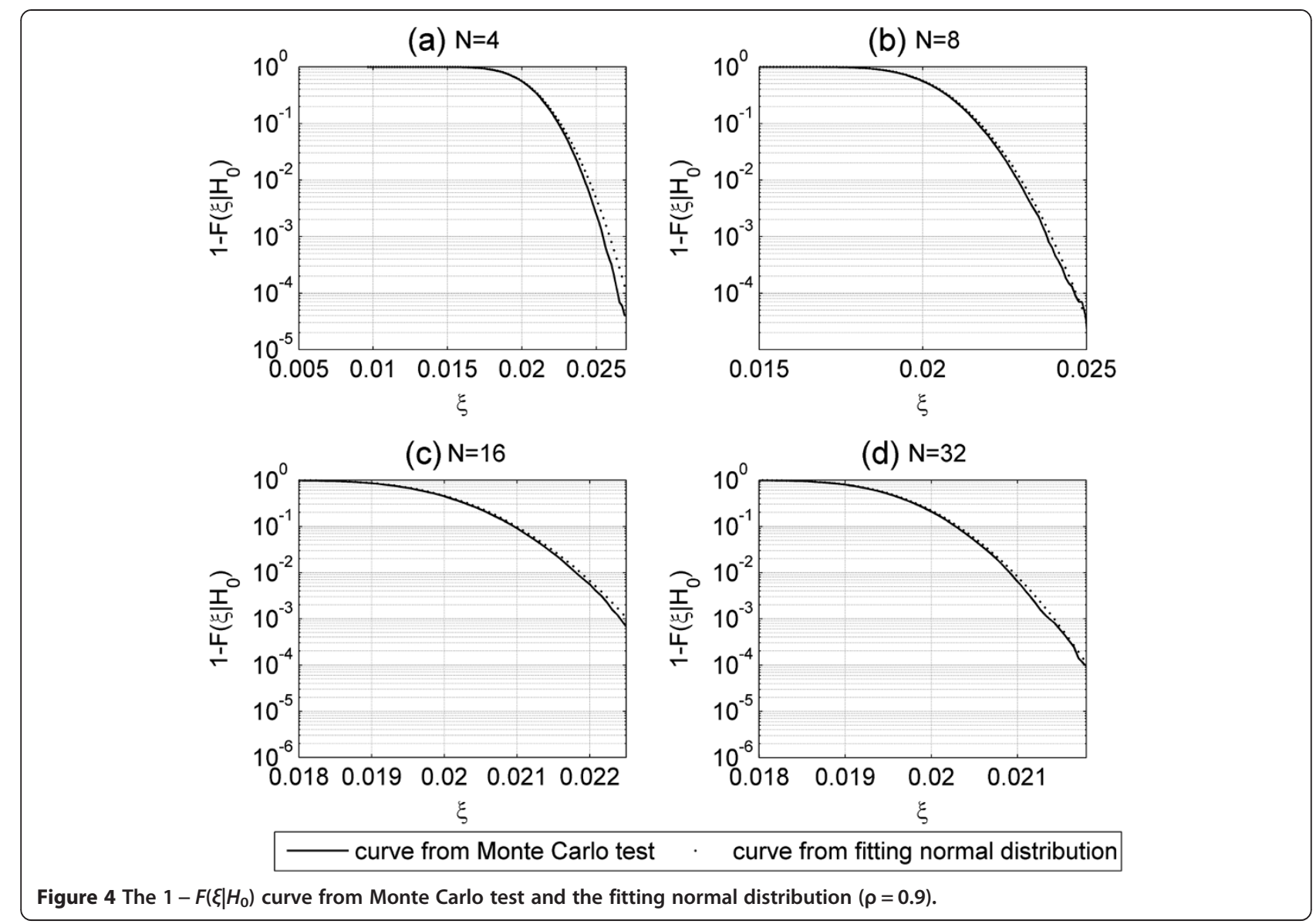

to different clutter level and clutter covariance matrices. Moreover, a specific nominal threshold $\eta$ is chosen to guarantee a predefined $\mathrm{P}_{\mathrm{fa}}$ which is selected from $1 \times 10^{6}$ Monte Carlo test. Then, the empirical $\mathrm{P}_{\mathrm{fa}}$ is the probability of the Gaussian random variable with fitting mean and variance larger than the specific nominal threshold $\eta$. Moreover, the empirical false alarm probability $P_{f a}=1-\Phi((\eta-\mu) / \sigma)$. In Figure 5, the empirical $P_{f a}$ with different integration number $N$ versus different one-lag correlation coefficient $\rho$ is shown. From this numerical experiment, we can find that the false alarm level is comparable with different clutter covariance matrices, so the proposed detector achieves an approximate constant false alarm ratio (CFAR) with respect to different clutter covariance matrices. Moreover, because the matching degree between empirical probability density function $p\left(\xi \mid H_{0}\right)$ and the fitting normal distribution becomes higher when integration pulse number $N$ increases, the difference between the empirical $P_{f a}$ and nominal $P_{f a}$ becomes smaller when $N$ becomes larger.

\section{Simulated results and performance assessment} 4.1. Weather clutter data simulation

For high range resolution weather clutter, the clutter samples at different range cells are assumed to be mutually independent. The clutter time series from a train of coherent pulses at each range cell is modeled as the product of a fast varying speckle component with Gaussian nature and a slowly varying spiky component with non-Gaussian nature. The correlation time of the speckle component

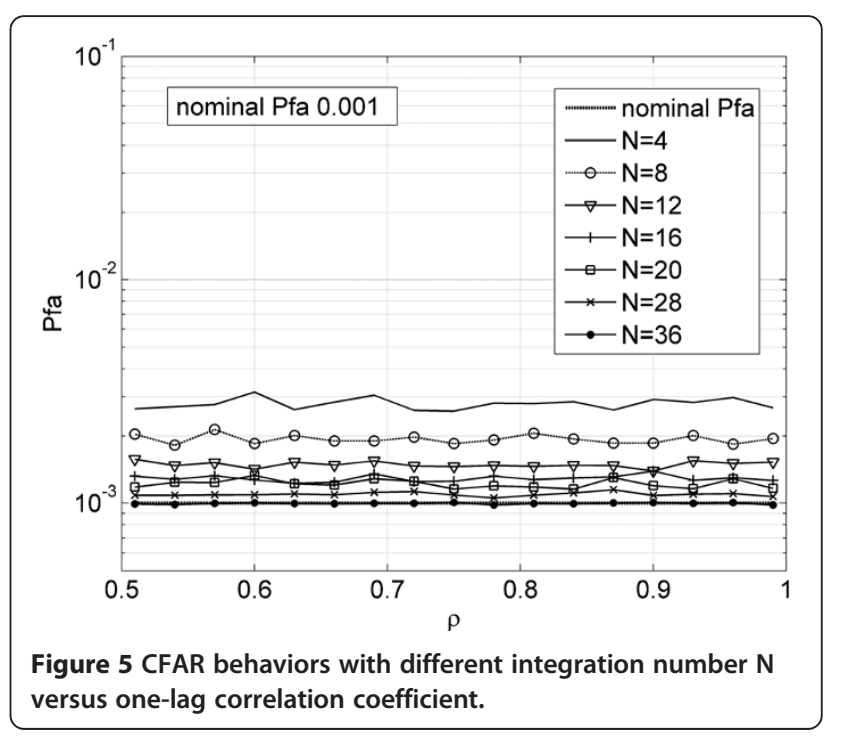


achieves tens of milliseconds and the spiky component exhibits a much longer decorrelation time. Hence, within the integration duration of tens of milliseconds, the clutter vector at each range cell can be modeled by a SIRV.

$$
\mathbf{c}_{m}=\sqrt{\mathbf{X}} \mathbf{u}_{m}
$$

where $\quad \mathbf{u}_{m}=[u(m, 0), u(m, 1), \cdots, u(m, N-1)] \quad$ is a $\quad N-$ dimensional zero-mean complex Gaussian random vector with the covariance matrix $\mathbf{R}$ whose diagonal entries are one and the positive random constant $X$ follows the Gamma distribution [10]:

$$
p_{X}(\chi)=\frac{1}{\Gamma(v)}\left(\frac{v}{\mu}\right)^{v} X^{\nu-1} e^{-\chi v / \mu}, X \geq 0
$$

In (24), $\Gamma($.$) is the Gamma function, \mu=E[\chi]$ is the mean of the random variable $\chi$, reflecting the average power of the clutter, and $v$ is a measure of clutter spikiness.

In terms of the basic assumptions in Section 2.1, the clutter vectors at all the range cells of the observation window of length $M+K$ share a same constant $X$ and a covariance matrix $\mathbf{R}$ of the speckle components. The weather clutter is a narrowband process and thus its covariance matrix is assumed as the form.

$$
[\mathbf{R}]_{i j}=\rho^{|i-j|}, \quad 1 \leq i, j \leq N
$$

where $\rho$ is one-lag correlation coefficient and its typical value is from 0.9 to 0.99 [21]. In each trial, we first generate a zero-mean complex Gaussian random matrix:

$$
\begin{aligned}
\mathbf{c}(m, n), m & =0,1, \cdots, M+K-1, \\
n & =0,1, \cdots, N-1,
\end{aligned}
$$

whose rows are mutually independent and each column has a covariance matrix $\mathbf{R}$ with the form in (25). Then, a positive random number $X$ followed the Gamma distribution is generated and the scalar product $\sqrt{X} \mathbf{c}(m, n)$ forms the clutter samples.

In experiments, raw target returns are added to the first $M$ range cells of simulated clutter data in terms of a predefined average signal-to-clutter ratio (A-SCR), which is defined as.

$$
\mathrm{A}-\mathrm{SCR}=10 \log _{10} \frac{\sum_{m=0}^{M-1} \mathbf{s}_{m}{ }^{H} \mathbf{s}_{m}}{M N \mu}
$$

where $\mu=E\{X\}$ is the average power of the clutter and the numerator is the total energy of target returns in the detection window. For a given A-SCR level and false alarm probability, when $Q$ independent trials of the raw target returns plus simulated clutter are tested, the detection probability is calculated by $Q_{\text {true }} / Q$, where $Q_{\text {true }}$ is the number of the true decisions.
The raw target data available, as shown in Figure 1, was collected from an aircraft in manoeuvring flight by a HRR at a good weather condition. Due to high SCR, the target data can be regarded to be free of clutter. Some sets of experiments are made for a full evaluation of the proposed detector.

\subsection{Performance of the proposed detector}

For moving target detection in the weather clutter, the Doppler bandwidth of the weather clutter has a strong impact on the detection performance. In the clutter model (24) and (25), the one-lag correlation coefficient $\rho$ determines the Doppler bandwidth of the clutter. The $\rho$ close to one corresponds to a narrow Doppler bandwidth of the clutter. For a range-spread target in highspeed manoeuvring flight, each scatterer of the target has generally a large Doppler shift. Due to their range walking across cells and rotation during integration, the target return at each range cell is the superimposition of several components with different Doppler shifts and different durations. When the Doppler bandwidth of the clutter is narrow, the temporal whitening preserves better target returns while the major clutter components in the low Doppler region are suppressed. Therefore, when $\rho$ is close to one, the proposed detector achieves better detection performance. In this experiment, we demonstrate the detection performance of the detector when $N=16$ and $\rho$ is sampled from 0.7 to 0.95 with an interval 0.05 . The length $M$ of the detection window is 128 . The number $K$ of the reference range cells is 512, which are located on the two sides of the detection window. In the simulation of the weather clutter, the parameters $\rho=0.9$, $\nu=0.5$ and $\mu=E\{X\}$ in the distribution of the random constant $X$ is specified by the given A-SCR. In the detection scheme, the temporal covariance matrix of the clutter vectors are estimated from the vectors at the reference range cells by the SCM estimator. In terms of the analysis of the raw target data, the maximal possible range walking $v_{\max } \mathrm{T}_{r} / \delta r$ between two adjacent pulses is taken as 0.5 . The false alarm probability is specified as 0.001 and the decision threshold is determined from the Monte-Carlo tests to clutter-only data. For each A-SCR level, 2000 independent trials are made to calculate the detection probability. As shown in Figure 6a, the detection performance rapidly becomes better as $\rho$ approximates to one.

Another factor to impact on the performance of the detector is the number $K$ of the reference range cells. As the number $K$ of i.i.d reference sample vectors increases, the estimated temporal covariance matrix is closer to the actual temporal covariance matrix. The approximate whitening filter approximates to the whitening filter and thus can better suppress the clutter. Therefore, with the increase of $K$, the detection performance of the detector 

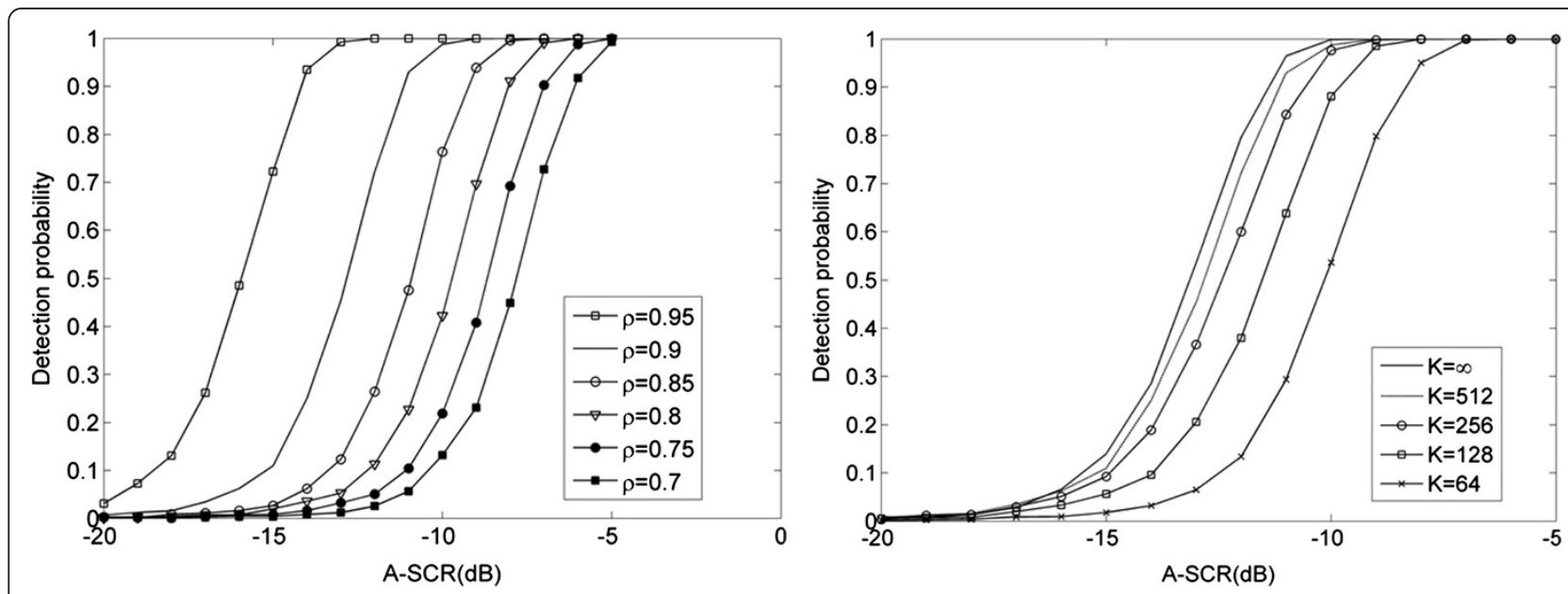

Figure 6 Performances of the proposed WCEN-based detector.

becomes better. In the second experiment, we demonstrate the detection performance when $N=16, K=64,128,256$, 512 , and infinite (corresponding to known temporal covariance matrix of the clutter), and $p=0.9, v=0.5$. The detection probabilities at these cases are shown in Figure 6b. It can be seen from Figure $6 \mathrm{~b}$ that the detection performance gradually becomes better as $K$ increases. In substance, the precision to estimate the temporal covariance matrix depends upon the ratio $K / N$. Moreover, we also find that when the ratio $K / N$ is no less than 16 , the performance of the detector is quite close to that of the detector with known temporal covariance matrix of the clutter vectors.

\subsection{Comparison with MCOM detector and GLRT detector}

The MCOM detector is a non-coherent detector for rangespread targets in white complex Gaussian noise in our previous work [5]. It consists of a nonlinear map on the amplitude-HRRPs for noise suppression and target return enhancement followed by a modified cross -correlation integrator. Here, we briefly review it. Let $\hat{z}(m, n), m=1,2$, $\cdots, M, n=1,2, \cdots, N$ be $N$ filtered amplitude-HRRPs by the nonlinear shrinkage map. Assume the maximally possible range walking of target during two adjacent pulses to be no more than $\Delta$ range cells. The modified crosscorrelation matrix (MCOM) of $N$ filtered amplitudeHRRPs is defined by.

$$
\begin{aligned}
& \boldsymbol{\Lambda}_{\mathrm{MCOM}} \equiv\left[\begin{array}{cccc}
\lambda_{\mathrm{MCOM}}(1,1) & \lambda_{\mathrm{MCOM}}(1,2) & \cdots & \lambda_{\mathrm{MCOM}}(1, N) \\
\lambda_{\mathrm{MCOM}}(2,1) & \lambda_{\mathrm{MCOM}}(2,2) & \cdots & \lambda_{\mathrm{MCOM}}(2, N) \\
\vdots & \vdots & \ddots & \vdots \\
\lambda_{\mathrm{MCOM}}(N, 1) & \lambda_{\mathrm{MCOM}}(N, 2) & \cdots & \lambda_{\mathrm{MCOM}}(N, N)
\end{array}\right] \\
& \lambda_{\mathrm{MCOM}}\left(n_{1}, n_{2}\right) \equiv \max _{|\tau| \leq \Delta\left|n_{1}-n_{2}\right|}\left\{\sum_{m} \hat{z}\left(m, n_{1}\right) \hat{z}\left(m+\tau, n_{2}\right)\right\} \text {. }
\end{aligned}
$$

where $\lambda_{\mathrm{MCOM}}\left(n_{1}, n_{2}\right)$ is the maximal value of the crosscorrelation function of two filtered amplitude-HRRPs within their maximally possible range walks, reflecting the energy and the waveform similarity of the two filtered amplitude-HRRPs. The conditional mean and variance of the random variable $\lambda_{\mathrm{MCOM}}\left(n_{1}, n_{2}\right)$ satisfy:

$$
\begin{aligned}
& E\left\{\lambda_{\mathrm{MCOM}}\left(n_{1}, n_{2}\right) \mid H_{0}\right\}=\sigma_{w}^{2} \kappa\left(\left|n_{1}-n_{2}\right|\right), \\
& \quad \operatorname{var}\left\{\lambda_{\mathrm{MCOM}}\left(n_{1}, n_{2}\right) \mid H_{0}\right\}=\sigma_{w}^{4} \rho^{2}\left(\left|n_{1}-n_{2}\right|\right),
\end{aligned}
$$

The qualities $\kappa(\bullet)$ and $\rho(\bullet)$ can be calculated offline from the Monte-Carlo tests to zero -mean unit-variance $\mathrm{M} \times \mathrm{N}$ noise matrices. The test statistic in the MCOM detector is

$$
\xi_{M C O M} \equiv \sum_{n_{1}=1}^{N} \sum_{n_{2}=n_{1}}^{N} \frac{\left\{\lambda_{\mathrm{MCOM}}\left(n_{1}, n_{2}\right)-\sigma_{w}^{2} \kappa\left(n_{2}-n_{1}\right)\right\}_{+}}{\sigma_{w}^{2} \rho\left(n_{2}-n_{1}\right)}
$$

where $\{x\}_{+}=\max \{x, 0\}$. According to the analysis above, both the MCOM detector and the WCEN-based detector exploit the energy and similarity of amplitudeHRRPs or power-HRRPs. Differently, the WCEN-based detector exploits the sparsity while the MCOM detector does not. This results in their difference in performance. Here, we use the MCOM detector, which combined with the prepositive approximate temporal whitening filter as does in the WCEN-based detector in the case of weather clutter, as a comparison with WCEN-based detector. In the experiments below, $\rho=0.9, v=0.5$, and the width of the detection window is $M=128$, the numbers of integrated pulses are $N=8,16$ and 32, the maximal possible range walking during two adjacent pulses is 0.5 range 
cell, and the false alarm probability is 0.001 . As shown in Figure 7a, the WCEN-based detector achieves much better performance than the MCOM detector does. This also shows that the sparsity is an important feature in detecting range-spread targets. In addition, it can be seen that doubling the number of integrated pulses brings about $1.5 \mathrm{~dB}$ A-SCR improvement for the proposed detector.

For range-spread targets in steady flight or in a short integration interval, the target returns can be modeled well by the simple tensor model in (4). Based upon that, the GLRT-based detectors [6] can coherently integrate target returns in non-Gaussian clutter for high performance detection. Target is assumed to have no aspect change and no range walking across cells during integration. Therefore, target returns are described as the tensor of an unknown complex vector and a Doppler steering vector with an unknown Doppler shift. The GLRT-based detector $[6,21]$ uses the test statistic:

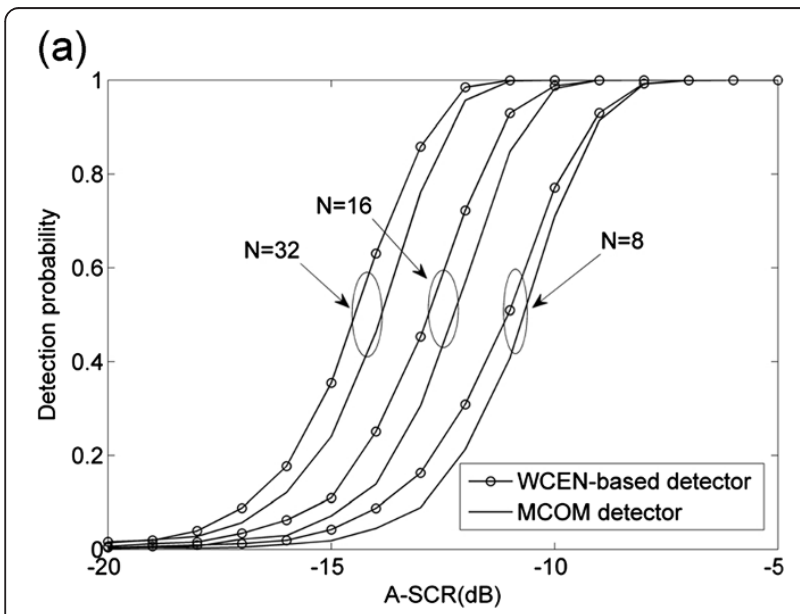

(b)

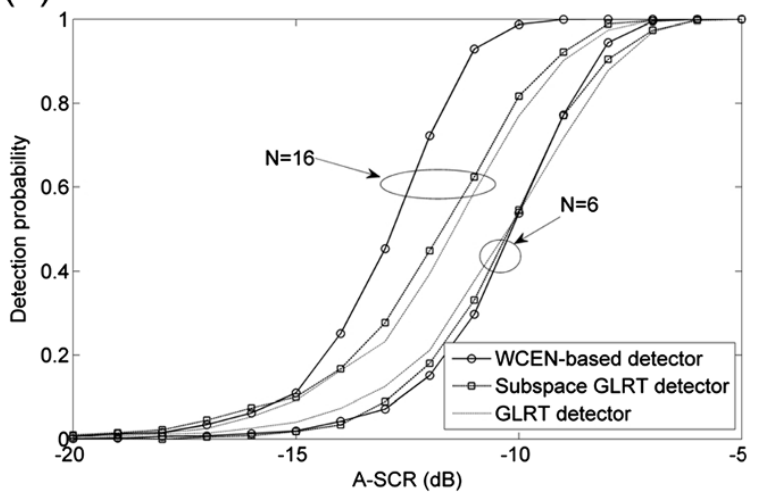

Figure 7 Comparisons of WCEN-based detector with some other conventional detectors. (a) Comparison of the WCEN-based detector and the MCOM detector (b) Comparisons of the WCEN-based detector, the GLRT detector and Subspace GLRT detector.

$$
\begin{aligned}
& \Lambda_{\mathrm{GLRT}}=-N \sum_{m=0}^{M-1} \ln \left(1-\frac{\left|\mathbf{p}^{H} \hat{\mathbf{R}}^{-1} \mathbf{z}_{m}\right|^{2}}{\left(\mathbf{p}^{H} \hat{\mathbf{R}}^{-1} \mathbf{p}\right)\left(\mathbf{z}_{m}{ }^{H} \hat{\mathbf{R}}^{-1} \mathbf{z}_{m}\right)}\right), \\
& \mathbf{p}=\left[1, \exp \left(2 \pi j T_{r} f_{d}\right), \exp \left(4 \pi j T_{r} f_{d}\right), \cdots, \exp \left(2(N-1) j T_{r} f_{d}\right]^{T},\right.
\end{aligned}
$$

where $\hat{\mathbf{R}}$ is the estimated clutter temporal covariance matrix from the secondary data and the Doppler frequency $f_{d}$ in the Doppler steering vector is estimated from the primary data. In the raw target data, range walking across cells rarely happens within six successive pulses. When $N=6$, target returns basically accord with the tensor model. When $N$ is large, for instance, $N=16$, the target returns do not meet the tensor model in [6,21]. In this case, the GLRT-based detector suffers from severe mismatch loss of target return model.

Furthermore, in order to account for the target Doppler spread in each bin, the subspace GLRT detector in [28] using the target model (5) described for homogeneous environment is considered. Here, $p_{m}$ in target model (5) is 2 . Then, the subspace GLRT detector is:

$$
\begin{aligned}
\Lambda_{\text {Subspace-GLRT }} & =-\sum_{m=0}^{M-1} \ln \left(1-\frac{\mathbf{z}_{m}^{\mathrm{H}} \mathbf{Q} \mathbf{z}_{m}}{\mathbf{z}_{m}^{\mathrm{H}} \hat{\mathbf{R}}^{-1} \mathbf{z}_{m}}\right), \\
\mathbf{Q} & =\hat{\mathbf{R}}^{-\mathbf{1}} \mathbf{U}\left(\mathbf{U}^{\mathrm{H}} \hat{\mathbf{R}}^{-\mathbf{1}} \mathbf{U}\right)^{-\mathbf{1}} \mathbf{U}^{\mathrm{H}} \hat{\mathbf{R}}^{-\mathbf{1}}
\end{aligned}
$$

where $\mathbf{U}$ is $N \times p_{m}$ matrix frequently called steering or mode matrix [28]. The target signal therefore belongs to the linear subspace spanned by the columns of the matrix U. Below, we compare the WCEN-based detector with the GLRT detector (31) and subspace GLRT detector (32) as $N=6$ and 16, where the clutter temporal covariance matrix is unknown and the number $K$ of the reference range cells is 512 . The one-lag correlation coefficient $\rho$ of the clutter is 0.9 . The false alarm probability is 0.001 and

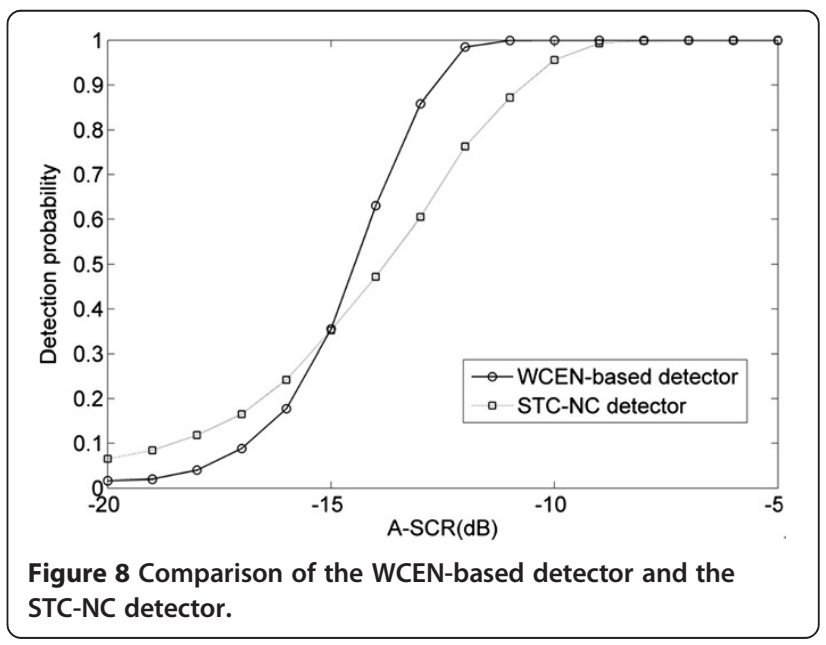


the maximal possible range walking between two adjacent pulses is 0.5 range cell in the WCEN-based detector. Their performance curves are shown in Figure 7b. When $N=6$, the GLRT detector and subspace GLRT detector are superior to the WCEN-based detector in the low A-SCR level. It is consistent with the common sense: the coherent integration generally outperforms the non-coherent integration as long as actual target returns meet the underlying signal model. When $N=16$, the WCEN-based detector much outperforms the GLRT-based detector and subspace GLRT detector, because the latter suffers from the severe loss of model mismatch. Moreover, because the model match-degree of subspace GLRT detector is higher than that of GLRT detector, the detection performance of subspace GLRT detector is better than GLRT detector.

Moreover, we consider another detector here. Precisely, based on the range migration estimation, the observation time can be divided into different sub-intervals where a coherent integration can be performed. Then, the different statistics, which are computed by the coherent integrations in each sub-interval, are incoherent integrated. And this detector can be called as shorttime coherent with long-time non-coherent (STC-NC) detector. In particular, the coherent statistic in each sub-interval can be computed by the subspace GLRT procedure in (32), and the non-coherent integration is the sum of the coherent statistics of all sub-intervals. And the final statistic of STC-NC detector is:

$$
\begin{aligned}
\Lambda_{\mathrm{STC}-\mathrm{NC}} & =-N_{s} \sum_{s=1}^{S} \sum_{m=0}^{M-1} \ln \left(1-\frac{\mathbf{z}_{m, s}^{\mathrm{H}} \mathbf{Q}_{s} \mathbf{z}_{m, s}}{\mathbf{z}_{m, s}^{\mathrm{H}} \hat{\mathbf{R}}_{s}^{-1} \mathbf{z}_{m, s}}\right), \\
\mathbf{Q}_{s} & =\hat{\mathbf{R}}_{s}^{-1} \mathbf{U}_{s}\left(\mathbf{U}_{s}^{\mathrm{H}} \hat{\mathbf{R}}_{s}-1 \mathbf{U}_{s}\right)^{-1} \mathbf{U}_{s}^{\mathrm{H}} \hat{\mathbf{R}}_{s}^{-1}
\end{aligned}
$$

where $\hat{\mathbf{R}}_{s}$ is the estimated clutter temporal covariance matrix in the $s$-th sub-interval, $N_{s}$ is the pulse number of the sub-interval. $\mathbf{U}_{s}$ is $\mathrm{N}_{\mathrm{s}} \times p_{m}$ matrix frequently called steering matrix of the $s$-th sub-interval. $\mathbf{z}_{m, s}$ is the received vector in the $m$-th range cell of the $s$-th sub-interval, and $S$ is the number of the subintervals. In the experiment, the pulses number $N_{s}$ of the sub-interval is set as 6 , based on the range migration estimation of the measured data. The number $S$ of the sub-intervals is 5 . For comparison, the integration number $N$ of the proposed WCEN-based detector is $N_{s} \times S=30$, which is the same as the total pulse number of the STC-NC detector. The one-lag correlation coefficient $\rho$ of the clutter is 0.9 . The false alarm probability is 0.001 and the maximal possible range walking between two adjacent pulses is 0.5 range cell in the WCEN-based detector. And the comparison result of WCEN-based detector and STC-
NC detector is shown in Figure 8. We find that the performance of WCEN-based detector is better than STC-NC detector in the high SCR environment and in the radar effective detection probability interval (generally, $P d \geq 0.8$ ). And in low SCR environment, the performance of WCEN-based detector is lower than STC$\mathrm{NC}$ detector. Therefore, from the above experiments in Figures 7 and 8, we can find that the WCEN-based detector is more suited to detect high-speed manoeuvring rangespread targets in a long integration interval.

\section{Conclusions}

This paper presented the WCEN-based detector for high-speed manoeuvring range-spread targets in weather clutter. It consists of the approximate temporal whitening filtering along pulses followed by the WCEN-based detection along range cells. The three inherent features, sparsity, similarity, and higher energy, are combined to design a test statistic for detection. The raw target data from a wideband HRR is used to fully evaluate the WCEN-based detector, showing it outperforms the MCOM detector, the GLRT detector, the subspace GLRT detector and the short-time coherent with longtime non-coherent integration detector in performance for high-speed manoeuvring range-spread targets.

\section{Competing interests}

Both authors declare that they have no competing interest.

\section{Acknowledgments}

This work was supported by National Natural Science Foundation of China (61201296, 61271024), the major program of National Natural Science Foundation of China (10990012), the Fundamental Research Funds for the Central Universities (K5051202037), and Guangxi Key Lab of Wireless Wideband Communication \&Signal Processing (12205).

The authors wish to thank the associate editor, reviewer 1, and reviewer 2 , who provided insightful comments and constructive criticisms that greatly improved the manuscript.

Received: 21 June 2012 Accepted: 18 March 2013

Published: 30 April 2013

\section{References}

1. A Gerara, S Van Der, Detection of a distributed target. IEEE Trans. Aerosp. Electron. Syst. 7(5), 922-931 (1971)

2. PK Hughes II, A high-resolution radar detection strategy. IEEE Trans. Aerosp. Electron. Syst. 19(5), 663-667 (1983)

3. HL Van Tress, Detection, Estimation, and modulation Theory Part III (John Wiley \& Sons. Inc, 2001). Chapter 12

4. K Gerlach, M Steiner, Detection of a spatially distributed target in white noise. IEEE Signal Processing Lett 4(7), 198-200 (1997)

5. PL Shui, SW Xu, HW Liu, Range-spread target detection using consecutive HRRPs. IEEE Trans. Aerosp. Electron. Syst. 47(1), 647-665 (2011)

6. K Gerlach, Spatially distributed target detection in Non-Gaussian clutter. IEEE Trans. Aerosp. Electron. Syst. 35(3), 926-934 (1999)

7. G Alfano, A De Maio, A Farina, Model-based adaptive detection of rangespread targets. IEE Proceedings of Radar, Sonar and Navigation 151(1), 2-9 (2004)

8. Y He, T Jian, F Su et al., Novel range-spread target detectors in nonGaussian clutter. IEEE Trans. Aerosp. Electron. Syst. 46(3), 1312-1328 (2010)

9. T Jian, Y He, F Su, C Qu, D Ping, Adaptive detection of sparsely distributed target in non-Gaussian clutter. IET Radar, Sonar and Navigation $5((7), 780-787$ (2011) 
10. N Bon, A Khenchaf, R Garello, GLRT subspace detection for range and Doppler distributed targets. IEEE Trans. Aerosp. Electron. Syst. 44(2), 678-696 (2008)

11. J Guan, XL Zhang, Subspace detection for range and Doppler distributed targets with Rao and Wald tests. Signal Process. 91(1), 51-60 (2011)

12. J Guan, YF Zhang, Y Huang, Adaptive subspace detection of rangedistributed target in compound-Gaussian clutter. Digit. signal process. 19(1), 66-78 (2009)

13. K Gerlach, MJ Steiner, Adaptive detection of range distributed targets. IEEE Trans. Signal Processing 47(7), 1844-1851 (1999)

14. E Conte, A De Maio, G Ricci, GLRT-based adaptive detection algorithms for range-spread targets. IEEE Trans. Signal Processing 49(7), 1336-1348 (2001)

15. A De Maio, A Farina, K Gerlach, Adaptive detection of range spread targets with orthogonal rejection. IEEE Trans. Aerosp. Electron. Syst. 43(2), 738-752 (2007)

16. L Du, HW Liu, Z Bao, JY Zhang, A two-distribution compounded statistical model for radar HRRP target recognition. IEEE Trans. Signal Processing 54(6), 2226-2238 (2006)

17. MD Xing, Z Bao, BN Pei, Properties of high resolution range profiles. Opt. Eng. 41(2), 493-504 (2002)

18. H Scott, P Demetri, Correlation filters for aircraft identification from radar range profiles. IEEE Trans. Aerosp. Electron. Syst. 29(3), 741-748 (1993)

19. C Chen, HC Andrews, Target motion induced radar imaging. IEEE Trans. Aerosp. Electron. Syst. 16(1), 2-14 (1980)

20. PL Shui, HW Liu, Z Bao, Range-spread target detection based on cross time-frequency distribution features of two adjacent received signals. IEEE Trans. Signal Processing 57(10), 3733-3745 (2009)

21. SG Jia, $\amalg$ Kong, A new approach to range spread target detection based on information entropy, Paper presented at The 2nd Asian-Pacific Conference on Synthetic Aperture Radar (IEEE press, Xi'an China, October 2009), pp. 560-562

22. SW Xu, PL Shui, XY Yan, CFAR detection of range-spread target in white Gaussian noise using waveform entropy. Electron. Lett. 46(9), 647-648 (2010)

23. XF Shuai, $\sqcup$ Kong, JY Yang, Performance analysis of GLRT-based adaptive detector for distributed targets in compound-Gaussian clutter. Signal Process. 90(1), 16-23 (2010)

24. E Conte, A De Maio, G Ricci, Recursive estimation of the covariance matrix of a compound-Gaussian process and its application to adaptive CFAR detection. IEEE Trans. Signal Processing 50(8), 1908-1915 (2002)

25. RS Murray, J Schiller, R Alu Srinivasan, Schaum's Outline of Theory and Problems of Probability and Statistics, Second Edition (McGraw-Hill Companies, 2002)

26. RR Coifman, MV Wickerhauser, Entropy-based algorithms for best basis selection. IEEE Trans. Inform. Theory 38(2), 713-718 (1992)

27. JD Echard, Estimation of radar detection and false alarm probabilities. IEEE Trans. Aerosp. Electron. Syst. 27(2), 255-260 (1991)

28. J Carretero, A De Maio, J Gismero, A Asensio, Experimental performance analysis of distributed target coherent radar detectors. IEEE Trans. Aerosp. Electron. Syst 48(3), 2216-2238 (2012)

doi:10.1186/1687-6180-2013-94

Cite this article as: $\mathrm{Xu}$ and Shui: Entropy-based detection of manoeuvring range-spread targets in homogeneous weather clutter. EURASIP Journal on Advances in Signal Processing 2013 2013:94.

\section{Submit your manuscript to a SpringerOpen ${ }^{\circ}$ journal and benefit from:}

- Convenient online submission

- Rigorous peer review

- Immediate publication on acceptance

- Open access: articles freely available online

- High visibility within the field

- Retaining the copyright to your article

Submit your next manuscript at $\gg$ springeropen.com 